\title{
Arrhythmogenic potential of antiemetics: perspectives on risk-benefits
}

\author{
Donald R. Miller MD FRCPC
}

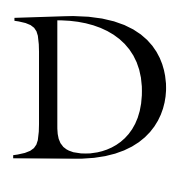

ROPERIDOL has been used for the management of postoperative nausea and vomiting (PONV) for over three decades. Relatively low doses of this drug (0.6-1.2 $\mathrm{mg} i v$ ) have been shown to be efficacious and costeffective as first- line therapy for PONV prophylaxis. ${ }^{1}$ However, a recent strengthening of the "black box" warning from the Food and Drug Administration (FDA) and Canadian Therapeutics Products Directorate regarding the arrhythmogenic potential of droperidol have resulted in many hospitals deleting this antiemetic from their drug formularies. The regulatory warnings emphasize that rare but significant cardiac morbidity and mortality are associated with droperidol, and that even low doses of the drug should be used only when other "first-line" drugs fail. ${ }^{2}$ The warnings that injectable droperidol should only be used in the hospital setting in order to allow for screening and monitoring of echocardiograms (ECG), that a 12-lead ECG must be done prior to drug administration to rule out prolonged QTc interval, and that the drug not be used as first-line therapy, will inevitably result in increased utilization of more expensive alternatives, despite rather limited and circumstantial evidence which led to the droperidol directives.

The droperidol warnings arose from reports that this drug causes QT prolongation and polymorphic ventricular tachycardia (Torsade de Pointes). While the cardiovascular effects of high doses of this drug have been recognized for some time, many anesthesiologists have questioned the evidence leading to a decision implicating the low doses used for antiemesis in the practice of anesthesia. Using the Freedom of Information Act, Bailey et al. recently provided a summary of the relevant cases which were reported to the FDA during a four-year time period. ${ }^{3}$ Of the 273 cases reporting adverse effects associated with droperidol, 127 serious adverse outcomes were identified in which patients experienced either death, prolonged hospitalization, or a life-threatening condition. However, a number of confounding factors were also identified, including amongst others, alcohol intoxication, suicide attempts, multi-organ dysfunction, and sepsis. Of 89 reported deaths, the dose of droperidol was $2.5 \mathrm{mg}$ or less in just two deaths; whereas the majority of deaths involved droperidol doses between 25-250 mg (ten to 100 times higher than doses generally administered during the course of anesthesia). A total of five patients receiving droperidol $2.5 \mathrm{mg}$ or less experienced either ventricular tachycardia or torsades with prolonged QT. Only one of these five cases was fatal. To date, there have been eight reported cases of sudden death associated with the use of droperidol with other agents in Canada. Interestingly, not a single case has been reported in the anesthesia literature of lowdose droperidol being associated with QTc prolongation, cardiac dysrhythmias or mortality in the perioperative setting.

The single most important observation from the currently available droperidol data is not that dysrhythmias occurred (the electrophysiological effects of the drug are well documented), but rather that the frequency of dysrhythmias using "antiemetic doses" is extremely low. The actual frequency is impossible to determine, but one might estimate that if $8 \%$ of the Canadian population undergoes an operative procedure each year, and that if $25 \%$ of these individuals received droperidol in a dose $<1.25 \mathrm{mg} i v$, the number of "treatments" per year would be in excess of one half million. The corresponding frequency of ventricular tachycardia or torsades would therefore be extremely low, probably be less than $0.0005 \%$. From the standpoint of patient safety, it is also important to consider that this drug is only administered in the perioperative setting in the presence of continuous electrocardiographic monitoring.

From the Department of Anesthesia, The Ottawa Hospital, Ottawa, Ontario, Canada.

Address correspondence to: Dr. Donald R. Miller, Department of Anesthesia, The Ottawa Hospital, General Campus, 501 Smyth Road, Ottawa, Ontario K1H 8L6, Canada. E-mail:dmiller@ottawahospital.on.ca

Reprints of this editorial will not be available from the author. 
Objections to the black box warning have been expressed, and others have recommended that the FDA lift the warning, and establish an expert advisory panel to review the reports and establish recommendations regarding the use of droperidol in the future. ${ }^{4}$ This is important because hospitals are naturally reluctant to expose themselves on medico-legal grounds so long as such warnings exist. In the meantime, two pharmaceutical companies have already discontinued their production of the drug.

As the fate of droperidol appears to have been established, it is important that anesthesiologists also be vigilant of rare but potentially important arrhythmogenic effects of more recently-introduced antiemetic medications. In this context, the report of new-onset atrial fibrillation following administration of ondansetron, which appears in this issue of The Journal, should be of interest to our readership. In their report, Kasinath et al. describe the events surrounding a 47-yr-old woman, free of cardiovascular disease, who underwent a breast lumpectomy under general anesthesia. ${ }^{5}$ The patient received ondansetron $4 \mathrm{mg} i v$ for PONV prophylaxis towards the end of the procedure, and an additional $4 \mathrm{mg} i v$ in the recovery room for nausea. Within 15 min of the second dose, her cardiac rhythm converted to atrial fibrillation, which required treatment with a procainamide infusion. The patient recovered uneventfully, and the authors postulated that ondansetron was the most likely cause of the fibrillation. While other causes of this arrhythmia could not be ruled out with certainty, the authors hypothesize that inhibition of $5-\mathrm{HT}_{3}$ receptors in the heart may have lead to unopposed action of other serotonin receptors, resulting in the new-onset atrial fibrillation.

The $5-\mathrm{HT}_{3}$ antagonists have been shown to block human cardiac $\mathrm{Na}^{+}$channels, which may lead to clinically relevant sodium channel block, especially in the setting of tachycardia or myocardial ischemia. ${ }^{6}$ Sodium channel blockade is associated with QRS widening, which may predispose to cardiac dysrhythmias. Ondansetron also possesses affinity for the $\mathrm{K}^{+}$ channel, which may prolong repolarization. None of the $5-\mathrm{HT}_{3}$ antagonists is thought to produce sufficient block of the slow delayed rectifier $\mathrm{K}^{+}$channel, which is implicated in the genesis of Torsade de Pointes. ${ }^{6}$ However, according to the ondansetron product monograph, there have been rare reports $(<$ $0.01 \%$ ) of various arrhythmias, including ventricular or supraventricular tachycardia, premature ventricular contractions, and atrial fibrillation, as well as second degree heart block associated with this drug (Zofran [package insert]. GlaxoSmithKline 2001).
Dolasetron is another $5-\mathrm{HT}_{3}$ antagonist which has been shown to be effective for both prophylaxis and treatment of PONV. Its primary clinical advantage is that a single dose may be effective for up to $24 \mathrm{hr}$ postoperatively. However, anesthesiologists should be aware of the fact that prescribing information for dolasetron includes a specific warning about the potential for QT interval prolongation and serious arrhythmias in at-risk patients (Anzemet [package insert]. Aventis Pharma 2002). Acute reversible ECG changes have been observed following administration of dolasetron. QTc prolongation may occur primarily due to QRS widening, but JT prolongation has also been observed. It has been established that the magnitude and frequency of ECG changes are related to the dose of the primary active metabolite, hydrodolasetron, and may persist for up to $24 \mathrm{hr}$. The Canadian product monograph also reports a case of complete heart block occurring intraoperatively in a 61-yr-old woman who received $200 \mathrm{mg}$ dolasetron orally for the prevention of PONV. This patient was incidentally also taking verapamil, which probably contributed to the atrio-ventricular (AV) conduction block. This emphasizes the importance of drug interactions in the perioperative setting. Due to established effects on cardiac conduction, the drug manufacturer (Aventis Pharma) has issued the following precautions: "Dolasetron should be administered with caution in patients who have or may develop prolongation of cardiac conduction intervals, particularly QTc due to potential additive effects. These include also patients with AV block II-III, bundle branch block, patients receiving concomitant class $I$ and III antiarrhythmics and patients with hypokalemia or hypomagnesemia, patients taking diuretics with potential for inducing electrolyte abnormalities, patients with congenital $Q T$ syndrome, patients taking antiarrhythmic drugs or other drugs which lead to QT prolongation, and cumulative high-dose anthracycline therapy. Interval prolongation could lead to cardiovascular consequences, including heart block or cardiac arrhythmias." Although the prescribing information for ondansetron does not contain a similar warning, there are cases reports of arrhythmias with ondansetron when this drug was given in conjunction with metoclopramide. ${ }^{7}$

It will be left to the reader to discern the probability of the causal relation of atrial fibrillation and ondansetron in the case report of Kasinath et al. ${ }^{5}$ In the evaluation of cause-effect relationships, it is interesting that this critical event occurred shortly after a second dose of $4 \mathrm{mg}$ (cumulative dose of $8 \mathrm{mg}$ ). Whether the event was idiosyncratic or dose-related cannot be established. When considering dose-response relationships 
and side effects, we should bear in mind the recent evidence suggesting that there is an absence of incremental therapeutic benefit with doses $>4 \mathrm{mg} i v$, and that repeat doses do not offer improved efficacy. ${ }^{8}$ The newonset atrial fibrillation does not appear to be related to an obvious drug interaction, as the patient was not receiving drugs which would interfere with cardiac conduction. Furthermore, there was no reason to suspect electrolyte abnormalities or cardiac conduction abnormalities in predisposing to this event. As cardiac dysrhythmias have been reported with both ondansetron and dolasetron, it is inevitable that the frequency of such reports will increase as their utilization in the perioperative setting increases.

Finally, the discussion would be incomplete without consideration of the efficacy of the antiemetic alternatives which have been shown to be cost-effective and free of associated arrhythmogenic effects. Dexamethasone $8 \mathrm{mg} i v$ can provide effective antiemetic prophylaxis for up to $24 \mathrm{hr} .{ }^{9}$ While it is relatively slow in onset and is most effective when used in combination with other antiemetics, it is also inexpensive ( $\$ 0.34$ per $8 \mathrm{mg}$ ). Prochlorperazine, a dopamine antagonist, has been shown to be equally efficacious when compared with ondansetron for single-agent antiemetic prophylaxis. This drug is also inexpensive at $\$ 0.32$ per 10 mg. ${ }^{10}$ In contrast, despite their long-standing use, the efficacy of dimenhydrinate and metoclopramide in the perioperative setting are somewhat disappointing. Prophylaxis of 15 adult patients with dimenhydrinate l-2 $\mathrm{mg} \cdot \mathrm{kg}^{-1}$ is required to prevent a single episode of PONV. ${ }^{11}$ Similarly, prophylaxis of ten adult patients with $10 \mathrm{mg} i v$ metoclopramide is required to prevent a single episode of PONV. ${ }^{12}$ Most studies show at least an additive effect from the use of two or more antiemetics (affecting different receptor types).

In conclusion, the recent and unfortunate fate of droperidol has resulted in a critical re-appraisal of alternative antiemetics for the perioperative setting. The 5-HT $\mathrm{H}_{3}$ antagonists ondansetron and dolasetron have been proven to be effective antiemetics, but we must also be mindful of rare but potentially serious adverse cardiovascular events associated with this group of drugs, as highlighted in this issue of The Journal. Clearly, there is no "magic bullet" for prevention and treatment of PONV. Efficacy, safety and side-effect profiles remain our primary concerns. The contemporary approach to prophylaxis and treatment of PONV remains: minimizing the doses of triggering agents in at-risk patients, using antiemetic drugs in combination, using lowest effective doses, and optimizing the timing of administration during the perioperative period.

\section{Le pouvoir arythmogène des antiémétiques : optique sur le rapport risques-bénéfices}

Le dropéridol est utilisé comme traitement des nausées et vomissements postopératoires (NVPO) depuis plus de trois décennies. On a montré que des doses relativement faibles de ce médicament $(0,6-1,2$ mg iv) étaient efficaces et économiques comme traitement de première intention pour la prévention des NVPO. ${ }^{1}$ Cependant, un renforcement récent de la mise en garde "boîte noire" de la Food and Drug Administration (FDA) et de la Direction des produits thérapeutiques canadiens concernant le potentiel arythmogène du dropéridol a amené de nombreux hôpitaux à retirer cet antiémétique de leurs formulaires. Les mises en garde des autorités soulignent que la morbidité et la mortalité cardiaques, rares mais significatives, sont associées au dropéridol et que même des doses faibles de ce médicament ne doivent être utilisées que lorsque d'autres médicaments de première intention n'ont pas eu d'effet. ${ }^{2}$ Les dispositions voulant que le dropéridol injectable ne soit utilisé que dans le milieu hospitalier pour permettre une sélection et une surveillance des échocardiogrammes (ECG), qu'un ECG à 12 dérivations devrait être fait avant l'administration du médicament pour exclure un intervalle QTc prolongé et que le médicament ne soit pas utilisé comme traitement de première intention, vont inévitablement provoquer une utilisation accrue d'autres médicaments plus chers, malgré une évidence plutôt limitée et circonstancielle qui a mené aux directives sur le dropéridol.

Les mises en garde relatives au dropéridol viennent d'articles montrant que ce médicament cause une prolongation du QT et une tachycardie ventriculaire polymorphe (Torsade de pointes). Même si les effets cardiovasculaires de fortes doses sont connus depuis un certain temps, de nombreux anesthésiologistes mettent en doute l'évidence qui a conduit à la décision sur les faibles doses utilisées comme antiémétique en anesthésie. En utilisant la Loi sur l'accès à l'information, Bailey et coll. ont récemment produit un résumé des cas pertinents rapportés à la FDA pendant quatre ans. ${ }^{3}$ Des 273 cas de complications associées au dropéridol, 127 complications sérieuses ont été iden- 
tifiées parmi lesquelles on note des décès, une hospitalisation prolongée ou un état critique. Cependant, un certain nombre de facteurs confondants ont aussi été identifiés, entre autres l'intoxication à l'alcool, des tentatives de suicide, une dysfonction multi-organique et de la septicémie. Dans les cas des 89 décès rapportés, la dose de dropéridol était de $2,5 \mathrm{mg}$ ou moins pour deux décès seulement tandis que dans la majorité des décès, les doses du médicament étaient de 25-250 $\mathrm{mg}$, c'est-à-dire de 10 à 100 fois plus que les doses généralement administrées pendant l'anesthésie. Cinq patients qui ont reçu $2,5 \mathrm{mg}$ de dropéridol, ou moins, ont été victimes d'une tachycardie ventriculaire ou des torsades avec un QT prolongé. Parmi ces cinq patients, un seul est décédé. Au Canada, on a rapporté jusqu'à maintenant huit cas de mort subite associée à l'usage de dropéridol combiné à d'autres médicaments. Fait à noter, aucun cas de faible dose de dropéridol associée à un QTc prolongé, à des dysrythmies cardiaques ou à de la mortalité périopératoire n'est mentionné dans la documentation anesthésique.

La seule observation d'importance provenant des données actuelles sur le dropéridol n'est pas que les dysrythmies peuvent survenir, les effets électrophysiologiques du médicament étant bien connus, mais plutôt que la fréquence des dysrythmies associées à des "doses antiémétiques" est extrêmement faible. La fréquence réelle est impossible à déterminer, mais on peut supposer que si $8 \%$ des Canadiens subissent une intervention chirurgicale chaque année et si $25 \%$ de ces individus reçoivent une dose de dropéridol $<1,25$ mg $i v$, le nombre de "traitements" annuels dépasse le demi-million. La fréquence de tachycardie ventriculaire ou de torsades correspondante serait donc très faible, probablement moins de 0,0005 \%. Du point de vue de la sécurité des patients, il est aussi important de dire que ce médicament n'est administré qu'en situation périopératoire en présence d'une surveillance électrocardiographique continue.

Des objections face aux mises en garde "boîte noire" ont été exprimées, d'autres ont recommandé que la FDA élimine la mise en garde, et qu'un groupe d'experts-conseils soit formé pour réexaminer les cas rapportés et mettre au point des recommandations sur l'usage futur du dropéridol. ${ }^{4}$ Cette démarche est importante, car les hôpitaux sont bien entendu réticents à s'exposer à des griefs médico-légaux aussi longtemps que les mises en garde sont en vigueur. En attendant, deux compagnies pharmaceutiques ont déjà abandonné la production du médicament.

Maintenant que le retrait du dropéridol semble accepté, il est important que les anesthésiologistes soient à l'affût des rares, mais potentiellement impor- tants, effets arythmogènes des médicaments antiémétiques récemment offerts. Dans ce contexte, l'article sur l'occurrence d'une fibrillation auriculaire de novo à la suite de l'administration d'ondansétron, qui paraît dans le présent numéro du Journal, devrait intéresser nos lecteurs. Dans leur article, Kasinath et coll. décrivent la situation d'une femme de 47 ans, sans maladie cardiovasculaire, qui a subi une tumorectomie du sein sous anesthésie générale. ${ }^{5}$ La patiente a reçu 4 mg d'ondansétron iv pour prévenir les NVPO jusqu'à la fin de l'opération, et une dose additionnelle de 4 mg iv à la salle de réveil pour les nausées. Moins de 15 min après la seconde dose, le rythme cardiaque s'est transformé en fibrillation auriculaire, ce qui a nécessité un traitement avec une perfusion de procaïnamide. La patiente s'est bien rétablie; les auteurs croient que l'ondansétron a été la cause la plus probable de la fibrillation. Même si d'autres causes de cette arythmie ne peuvent être exclues avec certitude, les auteurs émettent l'hypothèse que l'inhibition des récepteurs $5-\mathrm{HT}_{3}$ cardiaques peut avoir conduit à une action non compensée d'autres récepteurs de sérotonine, provoquant une fibrillation auriculaire de novo.

Les antagonistes de $5-\mathrm{HT}_{3}$ bloquent les canaux $\mathrm{Na}^{+}$ cardiaques chez l'humain, ce qui peut mener à un bloc cliniquement significatif des canaux sodiques surtout dans un contexte de tachycardie ou d'ischémie myocardique. ${ }^{6}$ Le blocage des canaux sodiques est associé à un QRS large qui peut prédisposer à des dysrythmies cardiaques. L'ondansétron possède aussi une affinité avec les canaux $\mathrm{K}^{+}$, ce qui peut prolonger la repolarisation. On croit qu'aucun des antagonistes de $5-\mathrm{HT}_{3}$ ne peut produire de blocage suffisant du canal $\mathrm{K}^{+}$lent rectificateur retardé, qui est impliqué dans la genèse de la torsade de pointes. ${ }^{6}$ Cependant, selon la monographie de l'ondansétron, il y a peu de cas (< $0,01 \%$ ) d'arythmies, y compris la tachycardie ventriculaire ou supraventriculaire, les extrasystoles ventriculaires et la fibrillation auriculaire, de même que le bloc cardiaque du deuxième degré associés à ce médicament (Zofran [notice d'accompagnement du produit]. GlaxoSmithKline 2001).

Le dolasétron est un autre antagoniste de $5-\mathrm{HT}_{3}$ qu'on sait efficace en prévention et en traitement des NVPO. Son principal avantage clinique est qu'une dose unique peut agir jusqu'à $24 \mathrm{~h}$ après l'opération. Toutefois, les anesthésiologistes ne doivent pas oublier que l'information posologique sur le dolasétron comporte une mise en garde spécifique sur la possibilité d'un allongement de l'intervalle QT et d'arythmies sérieuses chez les patients à haut risque (Anzemet [notice d'accompagnement du produit]. Aventis Pharma 2002). Des changements d'ECG brusques et 
réversibles ont été observés à la suite de l'administration de dolasétron. L'allongement de QTc peut se présenter surtout à cause de l'élargissement du complexe QRS, mais l'allongement de l'intervalle JT a aussi été noté déjà. Il a été démontré que l'importance et la fréquence des changements ECG sont reliées à la dose du principal métabolite actif, l'hydrodolasétron, et que ces modifications peuvent persister jusqu'à 24 h. La monographie canadienne des produits mentionne également un cas de bloc auriculo-ventriculaire complet survenu pendant l'opération chez une femme de 61 ans qui avait reçu $200 \mathrm{mg}$ de dolasétron oral pour prévenir les NVPO. Soit dit en passant, cette patiente prenait aussi du vérapamil, lequel a probablement contribué au bloc auriculo-ventriculaire (AV). Cette donnée souligne l'importance des interactions médicamenteuses pendant l'opération. Étant donné les effets connus sur la conduction cardiaque, le fabricant (Aventis Pharma) a émis les réserves suivantes : "Le dolasétron doit être administré avec prudence chez les patients qui ont manifesté, ou qui sont susceptibles de le faire, un allongement des intervalles de conduction cardiaque, surtout de l'intervalle QTc, causé par des effets additifs possibles. Ces patients sont aussi ceux qui présentent un bloc AV II-III ou un bloc de branche, ceux qui reçoivent en même temps des antiarythmiques de classe I et II et ceux qui souffrent d'bypokaliémie ou d'hypomagnésiémie, ceux qui prennent des diurétiques pouvant entraîner des déséquilibres électrolytiques, ceux qui présentent un syndrome congénital de l'intervalle QT, ceux qui prennent des antiarythmiques et de l'anthracycline à fortes doses cumulatives. L'allongement d'un intervalle peut avoir des répercussions cardiovasculaires, y compris un bloc cardiaque et des arythmies." Même si l'information posologique sur l'ondansétron ne contient pas de mise en garde semblable, certaines études de cas mentionnent des arythmies avec l'ondansétron quand ce médicament est administré avec du métoclopramide. ${ }^{7}$

Nous laissons au lecteur le soin de découvrir la relation causale possible entre la fibrillation auriculaire et l'ondansétron dans l'article de Kasinath et coll. ${ }^{5}$ Dans l'évaluation des relations de cause à effet, il est intéressant de noter que cet événement critique est survenu peu après une seconde dose de $4 \mathrm{mg}$ (dose cumulative de $8 \mathrm{mg}$ ). Que l'événement soit idiosyncrasique ou relié à la dose, on ne peut le déterminer. Quand on examine les relations doses-réponses et les effets secondaires, on doit penser à la récente preuve suggérant qu'il y ait une absence de bénéfice thérapeutique progressif pour des doses $>4 \mathrm{mg}$ iv et que des doses répétées n'offrent pas de meilleure efficacité. ${ }^{8} \mathrm{La}$ survenue d'une fibrillation auriculaire de novo ne semble pas reliée à une interaction médicamenteuse évidente, car le patient ne recevait pas de médicaments qui pouvaient nuire à la conduction cardiaque. De plus, il n'y a pas de raison de soupçonner les anomalies électrolytiques ou de la conduction cardiaque dans la prédisposition à cet incident. Des dysrythmies cardiaques ayant été rapportées avec l'ondansétron et le dolasétron, il est inévitable que la fréquence d'articles du genre augmente avec une plus grande utilisation périopératoire.

Finalement, la discussion ne serait pas complète sans parler de l'efficacité d'autres antiémétiques économiques et libres d'effets arythmogènes associés. Une dose de $8 \mathrm{mg}$ iv de dexaméthasone peut être une mesure prophylactique antiémétique qui se prolonge jusqu'à 24 h. ${ }^{9}$ Relativement lent à s'installer et plus efficace quand on l'utilise en combinaison avec d'autres antiémétiques, il est aussi bon marché $(0,34 \$$ les $8 \mathrm{mg}$ ). La prochlorpérazine, un antagoniste de la dopamine, est également efficace, comparée à l'ondansétron, comme antiémétique employé seul en prévention. Ce médicament est aussi économique à 0,32 \$ les 10 mg. ${ }^{10}$ Par ailleurs, malgré leur utilisation de longue date, l'efficacité périopératoire du diménhydrinate et du métoclopramide est plutôt décevante. Le traitement préventif de 15 adultes avec 1-2 $\mathrm{mg} \cdot \mathrm{kg}^{-1}$ de diménhydrinate est nécessaire pour empêcher un seul épisode de NVPO. ${ }^{11}$ De même, l'emploi de $10 \mathrm{mg} i \mathcal{v}$ de métoclopramide chez 10 adultes est nécessaire pour prévenir un seul épisode de NVPO. ${ }^{12}$ La plupart des études montrent au moins un effet additif de l'usage de deux antiémétiques ou plus affectant différents types de récepteurs.

En conclusion, la disparition récente et déplorable du dropéridol a entraîné la réévaluation critique d'autres antiémétiques périopératoires. L'ondansétron et le dolasétron, des antagonistes de $5-\mathrm{HT}_{3}$ se sont montrés d'efficaces antiémétiques, mais nous devons aussi demeurer conscients des rares, mais potentiellement dangereuses, complications cardiovasculaires associées à ce groupe de médicaments comme on le souligne dans le présent numéro du Journal. En clair, il n’y a pas de "potion magique" pour prévenir et traiter les NVPO. Les profils d'efficacité, d'innocuité et d'effets secondaires sont notre principal intérêt. L'approche contemporaine de la prévention et du traitement des NVPO demeure : la réduction des doses d'agents déclencheurs chez les patients à haut risque, l'utilisation d'antiémétiques en combinaison, l'utilisation des doses efficaces les plus faibles et la recherche constante du meilleur moment pour administrer le médicament pendant l'opération. 


\section{References}

I Watcha MF. The cost-effective management of postoperative nausea and vomiting (Editorial). Anesthesiology 2000; 92: 931-3.

2 www.fda.gov/bbs/topics/answers/2001/ans01123.html

3 Bailey P. The FDA droperidol warning: Is it justified?

(Letter) Anesthesiology 2002; 97: 288-9.

4 Tong JG, White PF, Scuderi PE, Watcha M, Kovac A. FDA "Black Box" warning regarding use of droperidol for postoperative nausea and vomiting: Is it justified? (Letter) Anesthesiology 2002; 97: 287.

5 Kasinath NS, Malak O, Tetzlaff J. Atrial fibrillation after ondansetron for the prevention and treatment of postoperative nausea and vomiting: a case report. Can J Anesth 2002; 50: 229-31.

6 Kuryshev YA, Brown AM, Wang L, Benedict CR, Rampe $D$. Interactions of the 5-hydroxytryptamine 3 antagonist class of antiemetic drugs with human cardiac ion channels. J Pharmacol Exp Ther 2000; 295: 614-20.

7 Baguley WA, Hay WT, Mackie KP, Cheney FW, Cullen $B F$. Cardiac dysrhythmias associated with the intravenous administration of ondansetron and metoclopramide. Anesth Analg 1997; 84: 1380-1.

8 Kovac AL, O'Connor TA, Pearman MH, et al. Efficacy of repeat intravenous dosing of ondansetron in controlling postoperative nausea and vomiting: a randomized, double-blind, placebo-controlled multicenter trial. J Clin Anesth 1999; 11: 453-9.

9 Henzi I, Walder B, Tramer MR. Dexamethasone for the prevention of postoperative nausea and vomiting: a quantitative systematic review. Anesth Analg 2000; 90: 186-94.

10 Chen JJ, Frame DG, White TJ. Efficacy of ondansetron and prochlorperazine for the prevention of postoperative nausea and vomiting after total hip or total knee replacement procedures: a randomized double-blind comparative trial. Arch Int Med 1999; 159: 615-21.

11 Kranke P, Morin AM, Roewer N, Eberbart LH.

Dimenhydrinate for prophylaxis of postoperative nausea and vomiting: a meta-analysis of randomized controlled trials. Acta Anaesthiol Scand 2002; 46: 238-44.

12 Henzi I, Walder B, Tramer MR. Metoclopramide in the prevention of postoperative nausea and vomiting: a quantitative systematic review of randomized, placebocontrolled studies. Br J Anaesth 1999; 83: 761-7. 